\title{
Acceptability of a role for community health workers in integrated mental health care for perinatal depression: a qualitative study from Surabaya, Indonesia
}

\author{
Endang Retno Surjaningrum ( $\nabla$ endang.surjaningrum@psikologi.unair.ac.id ) \\ Universitas Airlangga https://orcid.org/0000-0002-7555-7095 \\ Harry Minas \\ The University of Melbourne School of Population and Global Health \\ Anthony Francis Jorm \\ The University of Melbourne School of Population and Global Health \\ Ritsuko Kakuma \\ The University of Melbourne School of Population and Global Health
}

\section{Research}

Keywords: community health workers, integrated mental health care, perinatal depression, primary health care, Indonesia

Posted Date: March 12th, 2020

DOI: https://doi.org/10.21203/rs.3.rs-16915/v1

License: (1) This work is licensed under a Creative Commons Attribution 4.0 International License.

Read Full License 


\section{Abstract}

Background: Integrated antenatal care in Indonesia has the potential to extend the role of community health workers (CHWs) to include the identification of perinatal depression in the community and referral to primary health care $(\mathrm{PHC})$ centres, which is an example of task-sharing. This study aimed to examine the acceptability of this type of task-sharing in perinatal depression in Surabaya.

Methods: Semi-structured interviews were conducted with participants from four PHC stakeholder groups. 62 participants were recruited from $\mathrm{PHC}$ centres, villages and integrated health service posts, the district health office, and one hospital. Data were analysed using framework analysis.

Results: More than ninety percent of participants accepted or conditionally accepted task-sharing in perinatal depression. Acceptability was influenced by the perceived benefits of task-sharing, the convenience of the service delivery, the existence of cases of perinatal depression perceived by stakeholders, personal characteristics of $\mathrm{CHWs}$, and the scope of their responsibility within existing tasks. Acceptability was limited by stigma of mental health problems, confidentiality, CHWs' perceived selfcapacity, lack of confidence in CHWs' competencies, and the objectives of task-sharing in depression identification.

Conclusion: Results indicate the need to enhance $\mathrm{CHWs}$ ' knowledge about perinatal depression and the needs of users, and CHWs' competencies in identifying depression. Stigma influences potential health service users' attitudes to mental health services and help-seeking and help-providing behaviours among service providers, and can reduce motivation to use and to provide services.

\section{Background}

Worldwide, depression occurs in $12 \%$ of women in the perinatal period [1]. The prevalence of perinatal depression among women from low- and lower-middle-income countries has been reported to be 5-33\% during pregnancy and $5-60 \%$ postpartum [2]. A survey among postpartum mothers in Surabaya, Indonesia, found that the prevalence of depression was $22 \%$ [3]. Perinatal depression has an impact on mothers and their infants, affecting the mother-infant relationship, and resulting in reduction of breastfeeding, poor nutrition of mothers, and reduction in self-care among depressed mothers [4-6]. Depression during pregnancy may increase risk of intrauterine growth retardation, pre-eclampsia and rates of premature delivery [7].

Despite the prevalence and the impact of perinatal depression, screening for perinatal depression in routine care is not always available in low- and lower-middle-income countries, including in Indonesia. Nevertheless, antenatal care policies have adopted an integrated approach to enable depression to be identified and treated within primary health care (PHC). Such policies provide an opportunity for nonspecialist workers, e.g. community health workers (CHWs), to play an important role in the identification of depression among pregnant women and postpartum mothers, an example of task-sharing $[8,9]$. 
In the Indonesian health system, CHWs are community volunteers recruited from the community, who provide community-based maternal and child health services in integrated health service posts (ISPs) during an ISP monthly schedule of activity and home visits [10]. The ISP is a component of the primary health care network and is established in a village under the supervision of a PHC centre at the subdistrict level. It provides a basic health program, the main goal of which is to reduce infant mortality and to improve the health and nutritional status of children under 5 and pregnant and breastfeeding mothers [10-12]. A CHW may also carry out other tasks, including population and civil administration-related services [13], and environmental programs [14].

\subsection{Acceptability}

Padmanathan and De Silva [15] found that health service needs of the community and satisfaction with services contribute to acceptability of task-sharing in mental health care. Studies carried out in several countries have found that acceptability of task-sharing in mental health care is supported by perceptions of benefits such as accessibility, saving of time and money, and less stigma [16, 17]. Personal attributes of CHWs, such as gender, educational background, experience and knowledge, together with personal qualities (personality, being polite and friendly) also contribute to acceptability $[15,17]$. Proximity of nonspecialist health workers to service users, and community endorsement of their role, support community acceptability [17], whereas level of training, support and supervision are significant factors affecting acceptability among professionals [16]. From a health system perspective, acceptability is influenced by the availability of appropriate infrastructure, workload, and issues such as assurance of confidentiality [15]. Others reported that perception of CHWs as being trustworthy and as respected members of the community, and their links with the local health system, support acceptability of task-sharing in maternal mental health [17].

There is very little research examining the acceptability of task-sharing in identification of perinatal depression using WHO's health system framework as the basis. The framework considers the health system to be composed of six 'building blocks': service delivery; health workforce; information; medical products, vaccines and technologies; financing; and leadership and governance (stewardship) [18]. A report from the health system of Surabaya found that health system stakeholders consider task-sharing, specifically identification of perinatal depression by $\mathrm{CHWs}$, to be feasible in this context [19]. The goal of the present study is to examine whether PHC stakeholders in Surabaya consider task-sharing in identification of perinatal depression to be acceptable, using the WHO health system building blocks as an analytical framework.

\section{Methods}

\subsection{Research setting and participants}

The study was conducted in the PHC system in the city of Surabaya, Indonesia. Four groups of stakeholders participated: 1) service users - pregnant women and mothers up to one year postpartum, 2) health workers - GPs, nurses, midwives and counsellors in PHC centres, and CHWs from three villages, 3) 
mental health specialists, and 4) program managers from the district health office and those of ISPs from villages.

Three PHC centres that provide psychological services participated, from each of which health workers were recruited. From one of those centres, three villages were appointed to recruit ISP program managers, $\mathrm{CHWs}$, and service users. Two ISPs were selected from each village from which service users were recruited, as well as two out of five CHWs from each ISP, i.e. one CHW-manager and one CHW-member. Mental health specialists were recruited from the hospital. More details are available elsewhere [19].

\subsection{Data collection}

Participants' views on acceptability were collected through individual interviews carried out by the first author (ES). The interviews were conducted in Indonesian and, with the permission of participants, were audio-recorded. Participants were interviewed about their knowledge about and attitudes to mental health of mothers, their attitude to task-sharing in identification of perinatal depression, and their views concerning acceptability of this role for $\mathrm{CHWs}$. Participants were asked to consider two vignettes (Appendix I) briefly describing cases of perinatal depression as part of the interview focusing on participants' attitudes and the acceptability of a CHW role in the identification of perinatal depression. Participants were also informed about relevant policy statements [9] concerning the role of CHWs in the identification of perinatal depression prior to exploration in the interview of their attitudes concerning these statements.

\subsection{Data analysis}

Interview data (transcriptions of interviews) were managed using NVivo 11 and analysed, using framework analysis [20,21]. To improve the rigour of data analysis and prevent the loss of meaning, the data were analysed from the original language (Indonesian) which is the language of the analyser, and were translated into English when the analysis process moved to mapping and interpretation.

Two different analytic steps were applied. First, participants were categorised based on their level of acceptance to determine the distribution of responses among the participants. The responses were categorised on the basis of two criteria: (i) the acceptance of CHWs as non-mental health or non-health care professionals to carry out detection of depression and referral, and (ii) participants' acceptance that the identification could be conducted in ISPs. The four levels of acceptance were: 1) Acceptance, which refers to those who accepted both criteria; 2) Conditional Acceptance refers to participants who accepted both criteria but raised one or more requirements; 3 ) Non-acceptance refers to those who accepted neither the service delivery model nor CHWs as service providers; and 4) Uncertain, which is a classification for participants who were uncertain or did not state a clear view. Once respondents were categorised into the four groups, the transcripts were coded and charted using the WHO health system framework to explore the reasons behind acceptance/non-acceptance. Figure 1 illustrates the process of data analysis.

\subsection{Ethics Approval}


Ethics approval was obtained from the University of Melbourne on May 20 2015 (Ethics No. 1543833). Approval to conduct the research in Surabaya was granted by the Department of Health, Surabaya.

\section{Results}

\subsection{Participants}

Data collection took place between June and August 2015 by ES. The total number of participants in the study was 62 . Table 1 summarises the groups of participants and the numbers. It can be seen from the table that participants from service user groups comprised almost half. Of these, more than $80 \%$ had finished high school, but less than one-fifth were employed. Most participants were female, with only specialists and one health worker being male. Most participants came from the two main ethnic backgrounds, i.e. Javanese and Madurese. More details of the participants and their demographic features can be found elsewhere [19].

\subsection{Level of acceptance}

Almost three-quarters of participants $(n=45)$ accepted the proposed idea, while nearly one-fifth $(n=12)$ conditionally accepted the idea. Only $5 \%$ of participants $(n=3)$ were opposed and the rest $(n=2)$ did not state a clear view. Of those who accepted, $42 \%(n=26)$ were service users and $23 \%(n=14)$ were service providers. Most conditional acceptance $(n=11)$ came from professionals, i.e. service providers, specialists, and program managers, with only one service user in this category. Opposition came from two CHWs and a service user, with a strong voice of opposition from a CHW from a Madurese slum area. Figure 2 presents the proportion of stakeholders indicating acceptability. From the service providers group, about $60 \%(n=14)$ accepted the idea, nearly $30 \%(n=7)$ conditionally accepted, and two people (8\%) were opposed. One counsellor did not state her attitude clearly. Program managers were divided between the categories of acceptance $(60 \%, n=3)$ and conditional acceptance $(40 \%, n=2)$. For the specialists, the breakdown was: acceptance $40 \%(n=2)$, conditional acceptance $40 \%(n=2)$, and uncertain $20 \%(n=1)$. Service users overwhelmingly indicated acceptance $(90 \%, n=26)$, with only one person each indicating conditional acceptance and non-acceptance.

\subsection{Factors contributing to acceptability of task-sharing in perinatal depression}

There are several shared views among the groups of stakeholder on what makes task-sharing in perinatal depression identification acceptable or unacceptable, based on health system frameworks. Groups of participants have different views related to several health system areas, including service delivery, workforce, leadership/governance, and information systems, which contribute to acceptability. In contrast, privacy issues, stigma, and competencies of workforce were among the obstacles to tasksharing. Information systems, financing and some perceptions related to leadership and governance also limited acceptability. There were no views related to medical products and technologies. Figure 3 presents a summary of the study findings in the form of a health system matrix. 


\subsubsection{Service delivery}

Service delivery in the form of depression identification by $\mathrm{CHWs}$ was acceptable based on the benefits of the care and the convenience. However, stigma and privacy issues impede acceptability.

Women/mothers and CHWs perceived task-sharing as providing benefits to the health of mothers, and the physical, and developmental health of the infants. Beyond the role of $\mathrm{CHWs}$, depression identification among pregnant women and postpartum mothers could provide benefits to the mental health of the women and their babies. Mothers and CHWs believed that mentally healthy mothers would benefit the development of the infant during pregnancy and the relationship between mothers and their babies. In turn, this would prevent disruption to the development of the baby.

"it is important because mental health of mothers will influence the relationship between mothers and the baby" (Ppt E2)

Depression identification could improve the mental health of mothers in several ways: a more serious stage of depression could be prevented, and mothers feel their important role in raising children is recognized and appreciated. A mother of a seven-month baby described:

"the service is important because the health of both mother and child needs recognition. The mother who has maintained the health of the infant for nine months and then delivering it needs attention. But mostly, the attention is given only to the baby but not the mother. In fact, mothers need it, even though only in the way of asking a question about her feeling or situation, or a praise" (Ppt A2)

For young women in their first pregnancy, services provided by CHWs would enrich them with meaningful knowledge about the emotional life of mothers during pregnancy which otherwise they might not have. A young woman in her first pregnancy said:

"Sure, the mental health state of pregnant women is important. Sometimes a pregnant woman cries without reasons but is indeed questioning whether [the mood] will influence the infant inside, and at the end just try to ignore it...'I don't care'. That sometimes happens. "(Pr E2)

In addition, it could reduce anxiety regarding the delivery process. A young woman shared her story:

"...that would be good. It must be like that, indeed. There are pregnant women who are worried, feeling distress because they are afraid of delivering the baby and other causes. When I was in RKZ [hospital], a lot of young women shared such a story." (Pr C6)

Mothers also believed that the service has a good objective, which is helping women/mothers. The service could relieve the burden on a depressed mother or could be a first step towards getting other help, either from the CHWs or others.

As well as for service users, task-sharing could also provide benefits for CHWs, e.g. improving CHWs' knowledge and extending their skills regarding maternal and child health services. In addition, it could be 
of personal value to both $\mathrm{CHWs}$ and women/mothers. Mothers would be happy if there was chance to share their feelings, whereas $\mathrm{CHWs}$ viewed the new role as an opportunity to contribute to another social activity that they feel passionate about.

"I like to be involved in those sort of things..., assisting social works is one of my enjoyments." (CHW J4)

\subsubsection{The convenience of the service}

Task-sharing was seen as being convenient. The service is provided by $\mathrm{CHWs}$ who live in the same area as the users, and know and are known by the users. Health workers believed that living the same social and cultural life forms a strong social bond among them that results in mutual caring. In this way, they perceived that $\mathrm{CHWs}$ have cultural sensitivity that facilitates them approaching mothers in a more acceptable way compared to formal health workers.

"Because a CHW may already be known by the community, then her way to communicate, to be more open to the community is more acceptable than those of the formal health workers." (Mw 1)

Regular interaction also enables CHWs to be aware of the daily life of women in their area and therefore they could potentially recognize a mental health issue earlier if such a problem emerges.

"There is a CHW in every RT [an aggregate of a neighbourhood], therefore they will know things early" (Mw 3)

\subsubsection{Privacy issues and stigma towards mental health}

Privacy was a major concern in regard to the acceptable place where CHWs could carry on the task, whether at home through home visits or at an ISP during monthly maternal and child health services. Some women were worried about the presence of other family members if the care was provided at home, whereas other women felt uncomfortable if topics related to mental health or emotions were explored during an ISP activity. The ISP was seen as a place to talk about children's health rather than mothers'. It is also a crowded place where children are present as well as mothers and other women. On the other hand, a CHW may be reluctant to carry out the task at home because the 'home' is in fact a single room. The debate can be seen from these participants:

"Prefer during home visit. It is impossible to talk about my personal situation because the ISP is for children's health...it's better to visit home for that issue." (Ppt A3)

"Actually for CHWs it would be easier [to come] directly to the home. But for me, it is not comfortable [if it is conducted at home]" (Ppt C5)

"Can be approached during the ISP activity, because I cannot let myself enter her home, they rent only a small room for a whole family and the children behave" (CHW J3) 
Stigma also impeded the acceptability of task-sharing. Public stigma was raised by CHWs who described an existing view in the community that all issues in a family should be kept inside. These issues are not only about mental health, but also more general issues such as the lack of food in the family, resulting in the hiding of mental health issues from others. Many pregnant or postpartum women prefer to share their personal problems with family members or to go to professionals rather than to $\mathrm{CHWs}$.

"The demographic [structure] of the community make them think that [services provided by CHWs] are not important, that is how people here look at it. They also think that whether we have something to eat or not is our personal problem, let alone for mental health issues" (CHW M4)

Public stigma resulted in self-stigma when CHWs felt fear and insecurity about carrying out the task and led to opposition.

"If we are asked to explore deeper I don't agree [with doing the task] because it's too hard and risky, it is a personal matter and they may think that we want to get involved in it. If it's a health issue, then they can go to the PHC clinic" (CHW M1)

Because of public stigma, some women were reluctant to share their feelings to CHWs, since disclosure could generate negative feelings such as insecurity and shame.

"knowing each other [between users and CHWs] and disclosure of personal problems to neighbours generates insecurity and unease" (Ppt A2)

\subsubsection{Information systems}

Acceptability in regard to information systems was evident in a contradictory view about the existence of depression cases. Some groups of professional stakeholders, such as doctors, midwives, and specialists, believed that mothers with depression exist and that task-sharing is acceptable. In contrast, some CHWs perceived that depression cases do not exist among pregnant and postpartum mothers, leading to the view that the service is unnecessary. These differences could be best explained based on a general practitioner's illustration that the existence of cases is not always visible. Finding a depression case is difficult even for health professionals in a PHC centre, implying that a certain level of knowledge and skill is required. As a result, exact data about depression cases among pregnant and postpartum mothers is not available and the existence of cases is perceived differently among health workers. A doctor recalled his experience when he found indications of depression in two pregnant women when they were about to give birth. In fact, those women had attended regular pregnancy check-ups provided by health workers without any report of depression. Another practitioner described that a patient with depression sometimes would only be detected after the medical health assessment by a doctor failed to find any physical health problems despite multiple physical complaints given by the patient.

"So far, my understanding about perinatal depression... pregnant women who came to the centre complained things related to psychological issues. For example, we do not find hypertension but they keep complaining about headache even after being prescribed a medicine. Or they were anxious 
without knowing what makes their anxiety. Therefore, it seems like if we talk about exact data, depression data [based on diagnosis] cannot be found" (GP2)

Even though other health professionals and some $\mathrm{CHWs}$ also confirmed the existence of depression cases among pregnant women, they emphasized the scarcity of these cases. This belief was based on the absence of complaints from women, or if there were complaints, $\mathrm{CHWs}$ believed that these were not psychological issues.

\subsubsection{Health workforce}

In terms of health workforce, a role for CHWs was acceptable regarding personal characteristics and attributes of workforce, but their capability was questioned.

\subsubsection{Personal characteristics and attributes of workforce}

The concept of CHWs being the main workers in this role was seen in different ways by various groups of stakeholders. Mothers or women stressed personal qualities of the CHWs they worked with as a contributor. For example, a woman described a CHW as a kind and nice friend to talk to: "De ST is kind and nice as a friend to talk to, so it is okay" (Ppt A5). Another service user illustrated this by stating that CHWs are proactive people who usually show sensitivity to mothers' needs by proactively approaching mothers and stimulating a discussion about health. This strategy was responded to positively by mothers, when they shared their stories.

In addition to personal qualities, CHWs were perceived as having competencies to carry out the task because they have knowledge about and experience dealing with mental health issues. A CHW stated that they had experience handling cases of depression and other mental health problems among pregnant and postpartum mothers. This was strengthened by specialists and counsellors who explained that $\mathrm{CHWs}$ have been trained in mental health.

"The health office has provided training for CHWs for mental health. However, I think mere training is not enough; it needs to be continued with follow-up programs." (Cs 3 )

\subsubsection{Distrust of CHWs' competencies}

In contrast to their personal characteristics, the quality and competencies of $\mathrm{CHWs}$ in carrying out the task were questioned. Distrust of the competency of CHWs in identifying depression was raised by professional groups, viz. specialists, district program managers, and health workers. A district manager perceived $\mathrm{CHWs}$ as having limited capability; therefore, they can only carry on early detection and then must refer the woman to a midwife. Specialists also questioned $\mathrm{CHWs}^{\prime}$ competence in identifying depression symptoms. Even if CHWs agree to carry out the task, it should be adjusted to their capability, e.g. not making a judgement or diagnosis about the mental health status of a woman. A specialist said: 
"For early screening they need to be taught with the signs of depressed mothers in terms of physical, behaviour, and social [signs], but for confirming the diagnosis they have to refer them to professionals" (SP 5)

Health workers stressed the scarcity of CHWs who understand the signs of depression and could perform the task. They compared it to $\mathrm{CHWs}$ ' performance in administering a risk pregnancy scale, which not all $\mathrm{CHWs}$ are able to do. Parallel to the perceptions of professionals about them, some $\mathrm{CHWs}$ also perceived themselves to be incapable and preferred to not take on the task. This lack of self-confidence was mostly disclosed by CHWs-members and one of them even admitted that she does not know the signs that a pregnant woman is experiencing stress, expecting that she should not be assigned this role.

Besides the issue of competency in identification, some mothers raised their distrust in the capability of CHWs to maintain confidentiality. A mother explained:

"I am afraid that when I talk to someone [a CHW] about me, then she may talk to others. I would be ashamed" (Ppt A5)

\subsubsection{Financing}

Insufficient compensation was a reason for unacceptability. Despite the role of CHWs as volunteers, their role has been recognised and been rewarded with financial compensation. $\mathrm{CHWs}$ are provided with transport compensation to support their activities, including home visits. Nevertheless, for health workers, the amount of the rewards is very small and not equal to its burden and risks.

"[The task] is not hard, but please remember that working with the community needs financial support for transportation, and the amount is not significant" (Cs 3)

\subsubsection{Leadership/governance}

There were differences of opinion about whether depression identification is within CHWs' responsibilities in maternal and child health care. Expectations of the scaling up of the program to a national level, and negative beliefs about the task and the objective contributed to different opinions on acceptability.

\subsubsection{Task-sharing is CHWs' responsibility and involves expectations}

Acceptability of task-sharing was influenced by stakeholders' expectations of CHWs' responsibility. There were perceptions and expectations that depression identification is part of CHWs' existing tasks in maternal and child health care. For example, some CHWs believed that the task is within their responsibility, as one of them said straightforwardly: "It is indeed our task" (CHW J1). In addition, service users connected the task to their expectation about CHWs' role in providing help to mothers. A woman expected that: "It is the help that they should give anyway" (Pr C5).

In contrast, health workers and some other CHWs thought that the task was not the responsibility of CHWs for maternal and child health. Some health workers argued that if these CHWs are to be given the 
role, they should only detect the depression, but not take on other tasks such as recording, making reports, conducting formal assessments, and providing intervention. The task should also be embedded within their existing tasks and not be provided as a new and sole task. Despite providing them with these tasks, it was suggested that the recruitment of new $\mathrm{CHWs}$ should focus only on the detection of perinatal depression. A counsellor said:

"If they have to do reporting and filling in checklists it would be burdensome" (Cs3)

"Either the task: mental health, risk alert, infants and children's health, they [should] only report cases, not intervene" (Mw2)

In addition, some CHWs viewed the task as not only a burden for them but also put them in a risky situation since they must deal with family issues of service users. A CHW provided an illustration that depression among mothers may be caused by other family problems, such as domestic violence, in which they feel insecure about becoming involved.

"If we are asked to explore deeper I don't agree [to do the task] because it is too hard and risky, it is a personal matter and they may think that we want to get involved in. If it is a health issue, then they can go to the PHC centre" (CHW M1)

Moreover, task-sharing was expected to be a first step for a bigger goal that enables such services to be expanded into a national program. A centre manager shared her view:

"In fact, ... it is a good program that can be inserted [into the centre's program] so that it could be [adopted into] a national program" (GP)

\subsubsection{Unclear objective}

Objectives for the involvement of CHWs were questioned. Participants questioned how much follow-up could be done by a CHW after finding a mother who might be experiencing depression. For one mother, talking about her emotional problems with a professional such as a general practitioner or a gynaecologist was more meaningful, since they need to know about the infants' development and the mother's health, while CHWs could not approach it in a similar way. In other words, some participants needed to know the purpose of task-sharing that would make them use the service. A mother said:

"We feel reluctant because the objective is not clear. If it is a GP or a gynaecologist, they need to know the infant's development or the health status of mothers; while if CHWs know [the status] in detail they cannot do anything" (Ppt A2)

A similar concern was raised by a doctor who questioned the purpose of the role and saw a need for a legal foundation that stated the objective.

"CHWs will fill in the sheet by asking questions, indeed they need a legal foundation to ask them. [Other program] is set up to assess the risk of the delivery process; I want to know what this program 
will go for?" (GP1)

\section{Discussion}

This study aimed to examine the acceptability of task-sharing in the identification of perinatal depression from the perspectives of various health system stakeholders. The findings suggest that the women who are service users are the group with the highest percentage of acceptance (>90\%), yet this group also includes some who reject depression identification by $\mathrm{CHWs}$. Within the service providers' group, $\mathrm{CHWs}$ and health workers have a variety of positions, including those who accept, those who conditionally accept and those who strongly oppose. Stakeholder' perspectives generated a conclusion about the acceptability of task-sharing in the city of Surabaya that is consistent with the feasibility results [19].

Acceptability among stakeholders was viewed from the perspective of the benefits to mothers or women, and the convenience of service delivery. Participants' views on the quality or competence of CHWs indicated a debate that brings to light some issues with recruitment and training. Stigma and privacy issues are among the challenges to users and $\mathrm{CHWs}$ that need to be addressed. These issues are common to other areas of the health system, as are engagement with services, perception of the tasks, and increasing the competence of the workforce.

Perception of the benefits gained from task-sharing contributes significantly toward acceptability. In this study, formal workers and CHWs perceived that many different types of benefits would be gained when $\mathrm{CHWs}$ talk about depression to mothers/women. $\mathrm{CHWs}$ expect to get knowledge through training on this topic before carrying out the task. This knowledge would be beneficial to the establishment of a more accountable way to identify help needed by women so that early measures can be provided such as proper assessment and treatment from a specialist. Better mental health status of mothers would also benefit the health of infants. In addition to their professional life, the knowledge that CHWs gain through training about perinatal depression can also be applied to their personal life, such as caring the family. A similar finding related to the benefits of being a CHW has been reported by others [22]. This current finding calls for training of CHWs prior to carrying out the identification task, including the selection of training materials.

In addition, mothers feel that most of the attention regarding maternal care in the existing services is about the infants. Mothers perceived that their emotional needs during the maternal period have been neglected. A similar perception is also reported by women in high-income countries [23]. This current finding shows the underlying need to focus on women's mental health. It is recommended that CHWs acknowledge this focus and be equipped with a specific approach, for example by recognising and valuing the women's sacrifice for their maternal role.

Acceptability is influenced by perception of the existence of depression cases. There are two contradictory views: health workers believe that cases exist in the community, while some CHWs believe they do not. It seems likely that the inconsistency between these groups of stakeholders in looking at the existence of cases is because of differing competency in recognising symptoms of depression. 
Professionals such as mental health specialists and doctors can identify depression cases, since they have enough knowledge to see subtle forms of behaviour that indicate symptoms of depression. These participants are therefore sure that there are pregnant women who suffer from this disorder, regardless of whether the number is small or large. This perception contrasts with $\mathrm{CHWs}$ who are unsure about whether certain symptoms can be categorised as indicators of depression. These facts clearly indicate that $\mathrm{CHWs}$ lack competence in understanding the signs and symptoms of depression and recognising women who develop the disorder. Identifying signs and symptoms of depression is not an easy task, even for health staff [24]. However, not all CHWs may have the capacity to be trained, and not all of them may agree to participate. Indeed, improving knowledge could boost CHWs' confidence in taking on the role [16]. As well as being due to a lack of knowledge, $\mathrm{CHWs}$ ' lack of competence may be associated with a belief that depression is a 'normal' in pregnancy, as was reported by a participant in the study. This assumption is supported by the findings from an Australian community [25], however it needs to be examined within the Surabaya context. To improve CHWs' competence and the acceptability of task-sharing, training on perinatal depression for $\mathrm{CHWs}$ is required.

In addition, $\mathrm{CHWs}$ and professionals have different perspectives about the necessary numbers of cases as a basis to develop services. CHWs may be representing common views that a service is not required if cases are scarce, while professionals have a different view: no matter how small the number of people suffering from depression, care should be provided. Views about the right of people to get care and treatment for their mental health problem [26] is another significant reason that $\mathrm{CHWs}$, and the rest of the community need to be educated.

Stakeholders discussed CHWs' competence in keeping confidentiality and their insecurity about recording and sharing data on depressed mothers. On the one hand, women are afraid to disclose their personal problems to $\mathrm{CHWs}$, since they do not trust that $\mathrm{CHWs}$ could maintain confidentiality. On the other hand, CHWs may prefer not to know women's problems, as this could put them at risk for getting assault from the women's family if they want to help solve these problems. The situation is more complicated since CHWs are assigned to record data about women and households and share this for other purposes at the village level, such as for population and demographic input [14]. This complex role of CHWs enables women to trust them, but their role as an agent of the village in collecting, keeping, and sharing data needs to be addressed. A systematic review regarding the use of administrative data for research purposes [27] reported that acceptability toward the use of data for research is conditional on confidentiality, which cannot simply be ensured through anonymization. The need for consent for permission to use data by third parties is important. This review also found that primary health care providers, particularly those who are familiar to the community, have high levels of public trust. However, the review only mentioned health professionals, not volunteers. This debate implies a need to clarify the owner of the data: the health sector or the village. Data governance and a data sharing agreement should consider limited, de-identified, and sensitive data [28]. In regard to task-sharing in this current study, there is a need to train $\mathrm{CHWs}$ in the important aspects of data collection, including confidentiality and security of data, and to make explicit the relationship between data owners (service users) and collectors (CHWs). 
Stigma is a common issue related to mental health services. Task-sharing in mental health is generally challenged by public stigma that prevents people from seeking help [16, 29]. Stigma has also been reported by postpartum mothers as a problem [24] and a barrier to perinatal mental health care $[23,30]$. The current study suggests that public stigma is based on the community belief that mental health is a family issue that should not be disclosed to others, including to CHWs. A similar finding on the reluctance to discuss mental health issues because of family beliefs was reported from low income women in a large urban city in the US [31]. Another danger of stigmatizing beliefs in the community is the internalisation of public stigma to be self-stigma when people agree to the beliefs and apply it to themselves [32]. In this study, women/mothers internalise public beliefs by keeping personal problems to themselves or prefer to share them only with family members. These women/mothers also felt discomfort, shame and insecurity about disclosing personal problems to CHWs. Not only mothers or women, but $\mathrm{CHWs}$ also indicate stigmatizing attitudes by perceiving the role of task-sharing as a high-risk one, and their reluctance to participate in because it could increase tension between them and the mother's/women's family. Uncertainty and fear of the response from family, friends, and health professionals are also among the concerns about $\mathrm{CHWs}^{\prime}$ competence to keep confidentiality.

\subsection{Methodological considerations}

This qualitative study has a number of strengths. It is an attempt to collect data on stakeholders' perceptions of a program before it is even started. Since documents on the integration of mental health in maternal care are available, an investigation of perceptions is a necessary first step before implementation. The study was also conducted at the site where the infrastructure for the proposed program is available, i.e. where there are mental health specialists at a PHC. Unlike other studies in higher-income countries where referral becomes an issue [24], the research was carried out based on the consideration of the provision of referral within the PHC. Lastly, the study has successfully recruited a reasonable number of stakeholders from all levels of service providers and service users, as well as program managers from community and government institutions.

Despite these strengths, the study also has limitations. The study is a preliminary study before a maternal mental health care program has been developed, and motivated by policy documents that, to our knowledge, does not have an implementation plan. Further research is required to assess the implementation strategy and clarify some debatable issues.

\section{Conclusion}

Findings on the acceptability of task-sharing in integrated care for perinatal depression indicate the need to improve CHWs' knowledge and competencies in several aspects of mental health care. These are knowledge about perinatal depression including its symptoms, public beliefs about mental health, stigma, and confidentiality. These refer to elements of mental health literacy and indicate the need for educating $\mathrm{CHWs}$ about perinatal depression. Training $\mathrm{CHWs}$ in mental health literacy could potentially improve their ability to recognise mental health problems [33,34], decrease stigmatising attitudes, and 
increase confidence in providing help [35]. Similar training programs to those in other contexts are suggested for improving helping behaviour and help-seeking behaviour [36]. These connections lead to a recommendation to provide mental health literacy education about perinatal depression to CHWs, mothers, and related people in the community in Surabaya. Moreover, even though CHWs are not health professionals who are subject to professional ethics, educating them about confidentiality would protect CHWs as volunteers and mothers/women as health care users, and could improve the acceptability of task-sharing.

\section{Abbreviations}

WHO: World Health Organization; CHWs: community health workers; ISPs: integrated service posts; PHC: primary health care; GP: general practitioner; Ppt: postpartum; Pr: pregnant; Cs: counsellor; Mw: midwive; SP: service provider.

\section{Declarations}

The authors declare that the content of the manuscript has not been published, or submitted for publication elsewhere.

\section{Ethics approval and consent to participate}

Ethical approval for the study was obtained from the University of Melbourne in the Document No. 1543833. A research permit was released by Health Office of Surabaya. Informed consent was obtained from all participants prior to data collection.

\section{Consent for publication}

No applicable.

\section{Availability of data and materials}

The datasets used and/or analysed during the current study are available from the corresponding author on request.

\section{Competing interests}

The authors declare that they have no competing interests.

\section{Funding}

This study is part of the doctoral degree project conducted by the first author under supervision of the coauthors. There is no external funding to support the research

\section{Authors contributions}


ES conceived of the study, participated in its design and coordination, carried out the interviews and drafted the manuscript. RK, HM, and AJ guided ES in the conception and design of the study and supervised her work. RK also provided guidance on analysis and interpretation of the material. All authors read and approved the final manuscript.

\section{Acknowledgements}

We would like to thank to the Health Office of Surabaya and four PHC centres, three villages in Surabaya, and the Director of Dr. Soewandhi Hospital for providing permission and technical support. Our deepest gratitude goes to all participants whom we interviewed for this study, particularly the CHWs-manager who distributed letters of invitation to potential participants.

\section{Author details}

${ }^{1}$ Faculty of Psychology, Airlangga University, Indonesia

${ }^{2}$ Centre for Mental Health, Melbourne School of Population and Global Health, the University of Melbourne, Australia

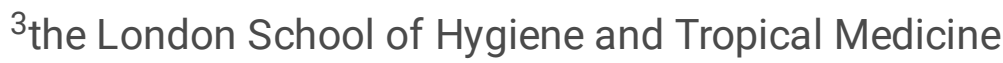

\section{References}

1. Segre, L.S., et al., The prevalence of postpartum depression. Social Psychiatry \& Psychiatric Epidemiology, 2007. 42(4): p. 316-321.

2. Fisher, J., et al., Prevalence and determinants of common perinatal mental disorders in women in low- and lower-middle-income countries: a systematic review. Bull World Health Organ, 2012. 90(2): p. 139G-149G.

3. Edwards, G.D., et al., Postnatal Depression in Surabaya, Indonesia. International Journal of Mental Health, 2006. 35(1): p. 62-74.

4. Flynn, H.A., Depression and postpartum disorders, in A public health perspective of women's mental health., B.L. Levin and M.A. Becker, Editors. 2010, Springer Science + Business Media: New York, NY, US. p. 109-120.

5. Bhutta, Z.A., et al., Global experience of community health workers for delivery of health related Millennium Development Goals: A systematic review, country case studies, and recommendations for integration into national health systems. 2010: World Health Orgnaization \& Global Health Workforce Alliance. 377-377.

6. Miranda, J.J. and V. Patel, Achieving the Millennium Development Goals: Does Mental Health Play a Role? PLoS Med, 2005. 2(10): p. e291.

7. Muzik, M., et al., When depression complicates childbearing: guidelines for screening and treatment during antenatal and postpartum obstetric care. Obstetrics \& Gynecology Clinics of North America, 
2009. 36(4): p. 771.

8. Ministry of Health Republic Indonesia, Guidance for Integrated Antenatal Care. 2010, Jakarta: Ministry of Health of Republic of Indonesia.

9. Ministry of Health Republic Indonesia, Manual Book for Cadres of Posyandu. 2012, Jakarta: Promotion Centre of the Ministry of Health of Republic of Indonesia.

10. Ministry of Health Republic Indonesia, Guideline for the Mangement of Posyandu. 2011, Jakarta: Ministry of Health Republic Indonesia.

11. Okamoto, A., Maternal and child health in Indonesia. Acta Paediatrica Japonica; Overseas Edition, 1993. 35(6): p. 559-566.

12. Andriani, H., C.-Y. Liao, and H.-W. Kuo, Association of Maternal and Child Health Center (Posyandu) Availability with Child Weight Status in Indonesia: A National Study. International Journal Of Environmental Research And Public Health, 2016. 13(3).

13. Ministry of Home Affairs, the Ministry of Home Affairs Regulation Number 28 Year 2011 about Guideline for FWM in Assisting Demographic Administration. 2011.

14. Ministry of Home Affairs, Decree of Ministry of Home Affairs Number 19 Year 2011 about Guideline for Integrated Social Services in Integrated Service Post 2011.

15. Padmanathan, P. and M.J. De Silva, The acceptability and feasibility of task-sharing for mental healthcare in low and middle income countries: A systematic review. Social Science \& Medicine, 2013. 97(0): p. 82-86.

16. Mendenhall, E., et al., Acceptability and feasibility of using non-specialist health workers to deliver mental health care: Stakeholder perceptions from the PRIME district sites in Ethiopia, India, Nepal, South Africa, and Uganda. Social Science \& Medicine, 2014. 118(0): p. 33-42.

17. Atif, N., et al., Barefoot therapists: Barriers and facilitators to delivering maternal mental health care through peer volunteers in Pakistan: A qualitative study. International Journal of Mental Health Systems, 2016. 10(1).

18. World Health Organization, Everybody bussiness: strengthening health systems to improve health outcomes: WHO's framework for action. 2007, Geneva: WHO.

19. Surjaningrum, E.R., et al., The feasibility of a role for community health workers in integrated mental health care for perinatal depression: a qualitative study from Surabaya, Indonesia. International Journal of Mental Health Systems, 2018. 12(1): p. 27.

20. Srivastava, A. and S.B. Thomson, Framework Analysis: A Qualitative Methodology for Applied Policy Research. Journal of Administration \& Governance, 2009. 4(2): p. 72-79.

21. Ritchie, J., et al., Qualitative Research Practice: A guide for social science students and researchers (2nd edition). 2014, London: Sage Publications Ltd.

22. Takasugi, T. and A.C.K. Lee, Why do community health workers volunteer? A qualitative study in Kenya. Public Health (Elsevier), 2012. 126(10): p. 839-845. 
23. Megnin-Viggars, O., et al., Experience of care for mental health problems in the antenatal or postnatal period for women in the UK: a systematic review and meta-synthesis of qualitative research. Archives of Women's Mental Health, 2015. 18(6): p. 745-759.

24. Tabb, K.M., et al., Perinatal depression screening in a Women, Infants, and Children (WIC) program: perception of feasibility and acceptability among a multidisciplinary staff. General Hospital Psychiatry, 2015. 37: p. 305-309.

25. Highet, N.J., A.W. Gemmill, and J. Milgrom, Depression in the perinatal period: awareness, attitudes and knowledge in the Australian population. Australian \& New Zealand Journal of Psychiatry, 2011. 45(3): p. 223-231.

26. Watters, C., Mental Health and IIIness as Human Rights Issues: Philosophical, Historical, and Social Perspectives and Controversies, in Mental Health and Human Rights: Vision, praxis, and courage, Michael Dudley, D. Silove, and F. Gale, Editors. 2012, Oxford University Press: Oxford.

27. Aitken, M., et al., Public responses to the sharing and linkage of health data for research purposes: a systematic review and thematic synthesis of qualitative studies. BMC Medical Ethics, 2016. 17(1): p. 73-73.

28. Allen, C., et al., Data Governance and Data Sharing Agreements for Community-Wide Health Information Exchange: Lessons from the Beacon Communities. eGEMS (Generating Evidence \& Methods to Improve Patient Outcomes), 2014. 2(1): p. 1.

29. Shakespeare, J., F. Blake, and J. Garcia, A qualitative study of the acceptability of routine screening of postnatal women using the Edinburgh Postnatal Depression Scale. The British Journal Of General Practice: The Journal Of The Royal College Of General Practitioners, 2003. 53(493): p. 614-619.

30. Nakku, J.E.M., et al., Perinatal mental health care in a rural African district, Uganda: a qualitative study of barriers, facilitators and needs. BMC Health Services Research, 2016(1).

31. Boyd, R.C., et al., Screening and Referral for Postpartum Depression among Low-Income Women: A Qualitative Perspective from Community Health Workers. Depression Research \& Treatment, 2011: p. 1-7.

32. Corrigan, P.W., et al., What is the impact of self-stigma? Loss of self-respect and the "why try" effect. Journal of Mental Health, 2016. 25(1): p. 10-15.

33. Armstrong, G., et al., A mental health training program for community health workers in India: impact on knowledge and attitudes. International Journal Of Mental Health Systems, 2011. 5(1): p. 17-17.

34. Murray, L.K., et al., A Common Elements Treatment Approach for Adult Mental Health Problems in Low- and Middle-Income Countries. Cognitive and Behavioral Practice, 2013(0).

35. Hofmann-Broussard, C., et al., A mental health training program for community health workers in India: impact on recognition of mental disorders, stigmatizing attitudes and confidence. International Journal of Culture \& Mental Health, 2017. 10(1): p. 62-74.

36. Patalay, P., et al., A Pre-Post Evaluation of OpenMinds: a Sustainable, Peer-Led Mental Health Literacy Programme in Universities and Secondary Schools. Prevention Science, 2017(8): p. 995. 


\section{Tables}

Tabel 1 Summary of participants by stakeholder group

\begin{tabular}{llr}
\hline Stakeholder group & Participants & $\mathbf{n}$ \\
\hline Service users & Pregnant women & 15 \\
Service providers & Postpartum mothers & 13 \\
& GPs & 3 \\
& Nurses & 3 \\
& Midwives & 3 \\
& Counsellors & 3 \\
Program Managers & CHWs-manager & 6 \\
& CHWs-member & 6 \\
Mental health specialists & District & 2 \\
Total & Community & 3 \\
\hline
\end{tabular}

\section{Figures}


Step 1

Level of acceptance
Step 2

Health system frameworks

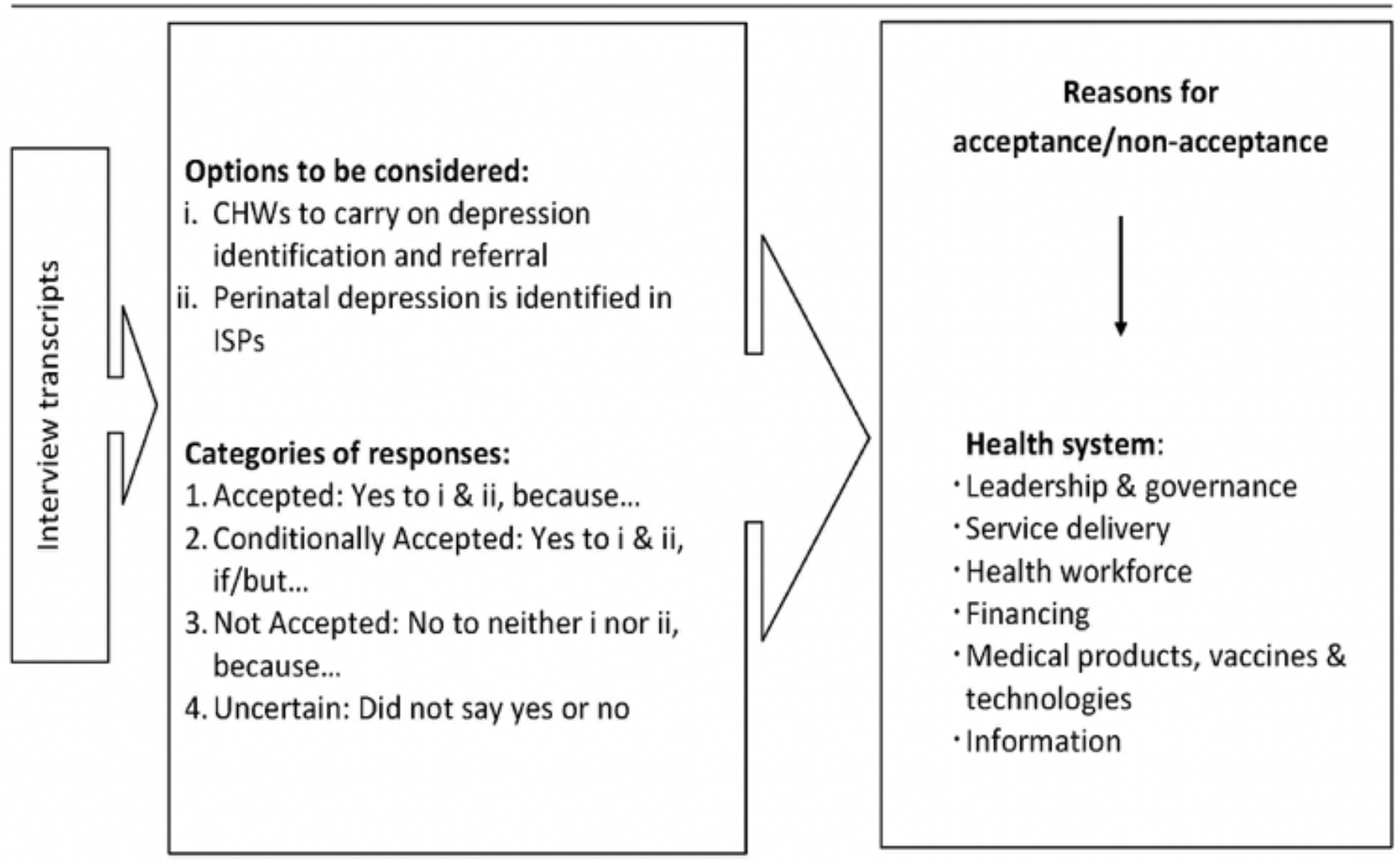

Figure 1

Flow diagram of data analysis 


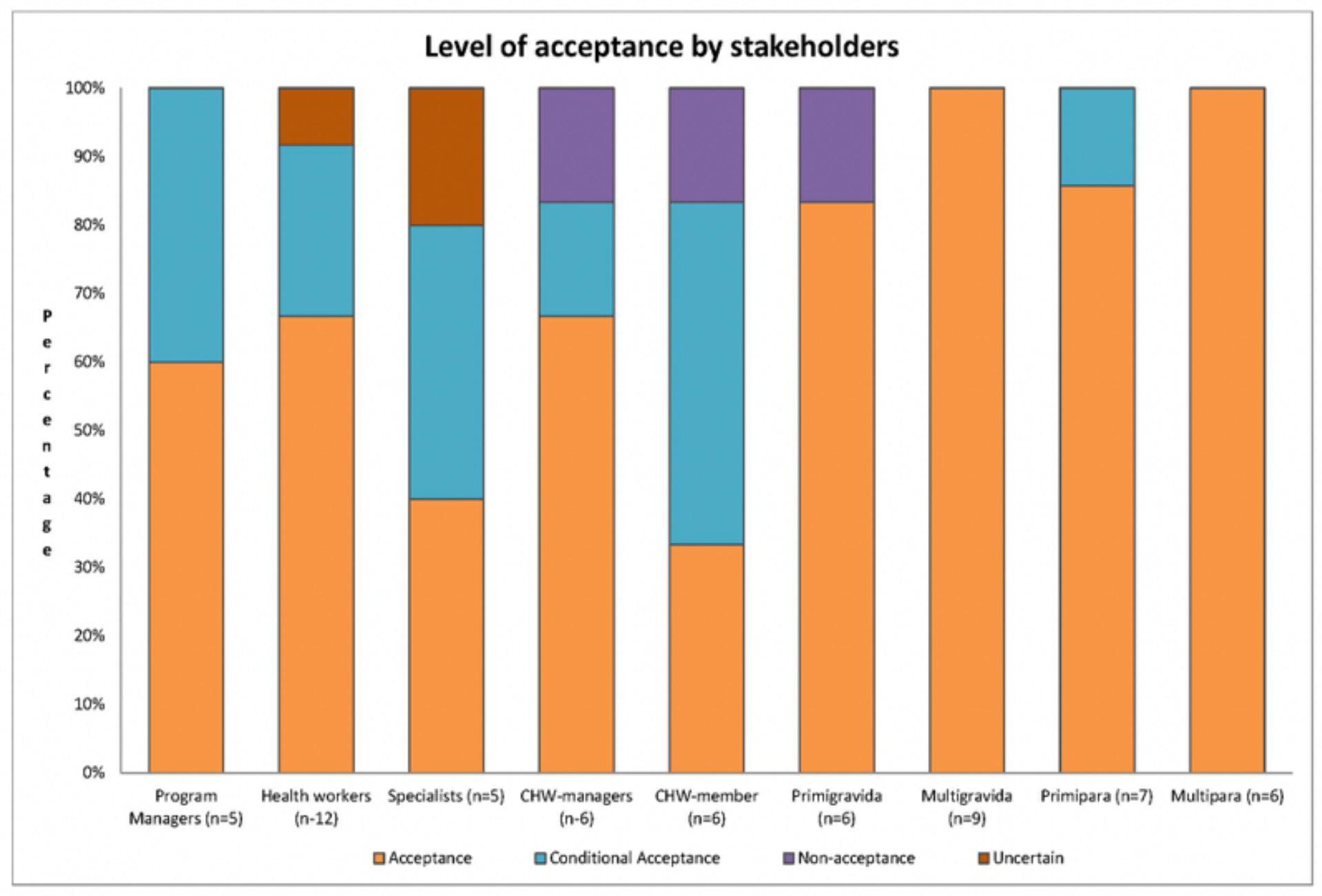

Figure 2

Proportions of participants' level of acceptance 
Factors contributing to lack of acceptability
Factors contributing

to acceptability
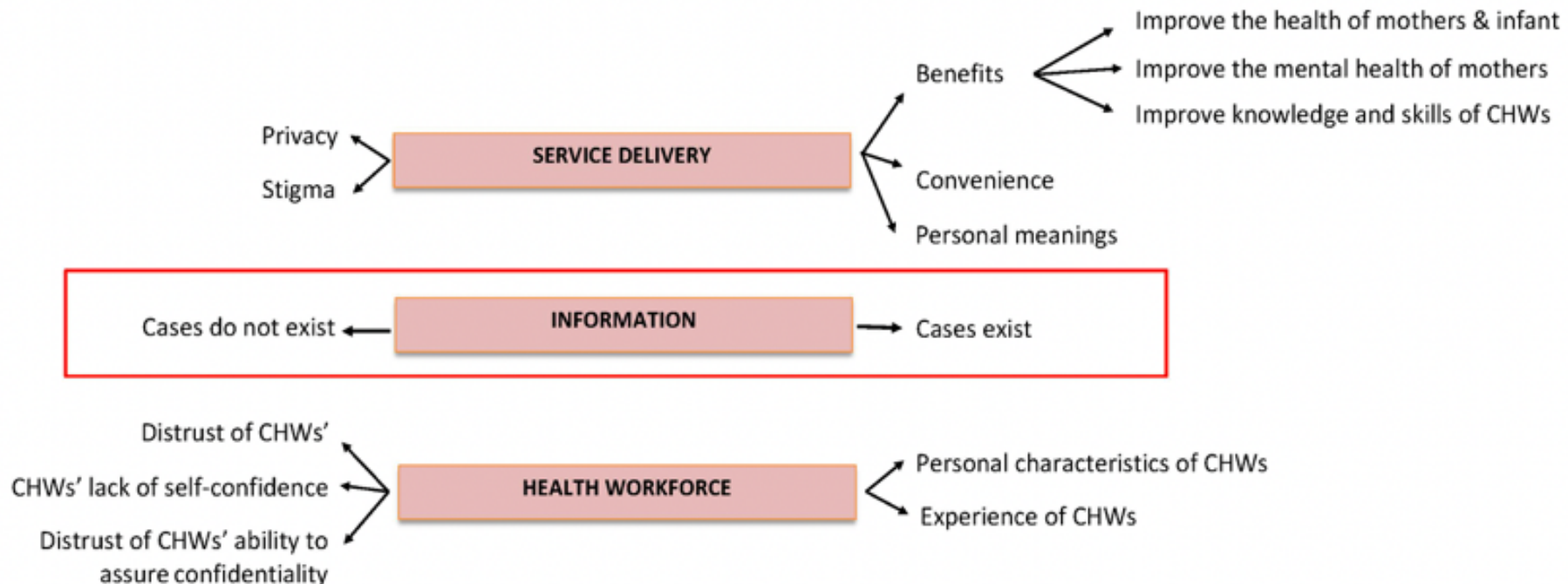

MEDICAL PRODUCTS \& TECHNOLOGIES

Insufficient

FINANCING

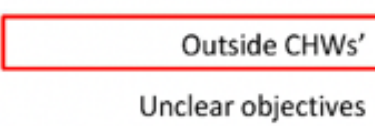

Negative beliefs about the
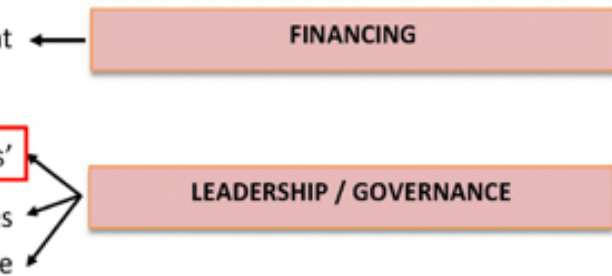

LEADERSHIP / GOVERNANCE

Within $\mathrm{CHWs}^{\prime}$ responsibility

There are inconsistent of perceptions

\section{Figure 3}

Summary of contributing factors to acceptability

\section{Supplementary Files}

This is a list of supplementary files associated with this preprint. Click to download.

- Appendix1Vignettas.docx 